\title{
Magneto-optical properties of the photon echo: status of research and prospects of application
}

\author{
I.I. Popov"1,*, A.U. Bahadurov'， N.S. Vashurin ${ }^{1}$ ， E.A. Vinogradov ${ }^{2}$, V.O. Kompanets ${ }^{2}$, \\ S.V. Chekalin', and S.E. Putilin ${ }^{3}$ \\ ${ }^{1}$ Volga State Technological University, 3 Lenin sq., Yoshkar-Ola, 424001 Russia \\ ${ }^{2}$ Institute of Spectroscopy of the Russian Academy of Sciences, 5 Fizicheskaya str, Moscow, Troitsk, \\ 108840 Russia \\ ${ }^{3}$ St. Petersburg National Research University of Information Technologies, Mechanics and Optics, \\ 49 Kronverksky Prospect, St. Petersburg, 197101 Russia
}

\begin{abstract}
The results of the study of the polarization properties of the stimulated photon echo (SPE) excited at room temperature on free excitons and exciton states localized on surface defects of textured thin films are reported. The results of observation and investigation of the effect of the non-Faraday rotation of a femtosecond SPE in a textured thin film are presented. The physical processes occurring under the Faraday effect and the effect of the non-Faraday rotation of the photon echo (PE) polarization plane are compared.
\end{abstract}

When the PE is formed in the presence of a longitudinal homogeneous magnetic field, the effect of the non-Faraday rotation of the PE polarization plane takes place. This effect differs fundamentally from the Faraday effect in the physics of the occurring processes and the magnitude of the rotation angle, which is three times bigger in exponent part than the Faraday rotation at the same values of the intensity of the longitudinally applied uniform magnetic field. The angle of the non-Faraday rotation of the PE polarization plane depends on the $\mathrm{g}$ factor of the quantum transition of the resonant medium, on the magnitude of the applied magnetic field $\mathrm{H}$ and on the time intervals separating the exciting pulses. The PE is formed during the optical transient process when the exciting laser radiation is applied to a resonant medium. The difference between these effects is as follows.

When the quantum system is excited by pulses with a duration much shorter than the relaxation times of the resonant medium equally polarized pseudoelectric dipoles are produced on all particles. The process of dephasing (phasing) of these dipoles occurs at different phase velocities for the upper (dextrorotatory) and lower (left-handed) components of the hyperfine structure of the excited level, which is split due to the Zeeman effect. The upper component increases, while the lower component decreases the phase velocity of the precession of the dipole. The resulting phase velocity difference, which leads to the rotation of the polarization plane of the echo signal, increases with increasing magnetic field strength and the time of dephasing (phasing) of the dipoles, given by the value of the time intervals

\footnotetext{
*Corresponding author: Popov@volgatech.net
} 
separating the exciting pulses. The non-Faraday rotation occurs both for the PE and for the SPE.

For the first time, the non-Faraday rotation of the SPE at room temperature in the nanosecond range of durations is performed in molecular iodine vapor [1]. Since we first obtained femtosecond signals of the primary photon echo PE and SPE on free excitons and excitons localized on surface defects assembled into nanoscale clusters, we had the opportunity to conduct a search for the non-Faraday rotation of the PE polarization plane in an exciton gas. Because of the high temperature, the thermal energy of the excitons is comparable to their binding energy, so a significant part of the excitons localized in the cluster recombine, and the concentration of the remaining excitons decreases to a concentration typical for the gas medium. This circumstance makes it possible to effectively use the available theory of the effect of the non-Faraday rotation of the polarization plane of the PE in a gas very effectively for processing the experimental results obtained. The difference in the defects entering the cluster ensures an inhomogeneous broadening of the exciton gas, which is necessary for the formation of a photon echo. The photon echo is formed during the transient process of formation of the exciton state, that is, on superposition states of pseudoelectric dipoles created by an electron having a Coulomb binding with a semiconductor hole.

A series of our experiments with femtosecond pulses on the study of the polarization properties of SPE in the presence of a longitudinal homogeneous magnetic field includes:

1. Detection of the effect of the non-Faraday rotation of the polarization plane of the SPE.

2. The results of the research of this effect allow to register the femtosecond time intervals at room temperature on-line.

3. Detection of the inhomogeneous distribution of localized exciton states by measuring the $g$ factor of quantum transitions from the repetition period of the modulation on the slope of the SPE curve as a function of the time interval between the exciting pulses of $\tau_{23}$ for several discrete values of the time interval $\tau_{12}$. These results can be used as the basis for developing a new method of femtosecond magneto-optical spectroscopy based on SPE.

4. Detection of a long-lived femtosecond PE on localized exciton states at room temperature.

The principal difference between the last experiment on long-lived memory in the works of I.A. Akimov and his coauthors [2,3] is the following (apart from the fact that the colleagues observed a nanosecond $\mathrm{PE}$ at helium temperature, and not a femtosecond PE, like us, and at room temperature). In their works, the Larmor precession under the action of a transverse magnetic field was used to switch over the back of the object on which the PE was formed. In our research, the Larmor precession, under the action of a longitudinal magnetic field, influenced the phase velocity of the precession of the pseudoelectric dipole with a different contribution for the components of the hyperfine structure of the excited quantum level, from which the degeneracy was removed due to Zeeman splitting.

\section{References}

[1] I.S. Bikbov, I.I. Popov, V.V. Samartsev, I.V. Yevseyev, Laser Phys. 5(3), 580 (1995)

[2] L. Langer, S.V. Poltavtsev, I.A. Yugova et al., Phys. Rev. Lett. 109, 157403 (2012)

[3] L. Langer, S.V. Poltavtsev, I.A. Yugova et al., Nature Photon. 8, 851 (2014) 